\title{
Clock Constraint Specification Language Specifying Clock Constraints with UML/MARTE
}

\author{
Frédéric Mallet
}

Received: 1 July 2008 / Accepted: 26 July 2008 / Published online: 9 August 2008

\begin{abstract}
The OMG UML Profile for Modeling and Analysis of Real-Time and Embedded systems (MARTE) aims at using the general-purpose modeling language UML in the domain of Real-Time and Embedded (RTE) systems. To achieve this goal, it is absolutely required to introduce inside the mainly untimed UML an unambiguous time structure which MARTE model elements can rely on to build precise models amenable to formal analysis. MARTE Time model has defined such a structure. We have also defined a non-normative concrete syntax called CCSL to demonstrate what can be done based on this structure. This paper gives a brief overview of this syntax and its formal semantics and shows how existing UML model elements can be used to apply this syntax in a graphical way and benefit from the semantics.
\end{abstract}

Keywords Unified Modeling Language · Time model · Constraints

\section{Introduction}

The Unified Modeling Language (UML) [6] aims at being a unified and general-purpose modeling language. Its semantics is purposely loose to cover a large domain and introduces so-called semantic variation points that provide for extensions to refine (or even define) a semantics when required for a specific domain. These extensions are to be defined in the context of a UML Profile. In the domain of real-time and embedded (RTE) systems, the Object Management Group (OMG) has recently adopted the UML Profile for Modeling and Analysis of Real-Time and Embedded systems (MARTE) [7], which is currently in the finalization phase. In its foundations, MARTE defines a broadly expressive Time Model to provide for a generic timed interpretation of UML models. The idea is to precisely define a semantics within the Profile rather than allowing tools for giving their own, possibly incompatible with other tools of the same domain.

F. Mallet

Université de Nice Sophia Antipolis

INRIA Sophia Antipolis Méditerranée

2004 rte des Lucioles - 06903 Sophia Antipolis cedex - FRANCE Tel.: +33-4-9238-7966

E-mail: fmallet@sophia.inria.fr 
MARTE Time Structure is heavily inspired by the Tagged Signal Model [4], which intends to define a common framework for comparing several Models of Computation and Communication in the RTE domain, and from various works around synchronous languages [3] and more generally polychronous/multiclock languages well-suited to specify Globally Asynchronous and Locally Synchronous (GALS) systems. The concrete syntax of our language, called Clock Constraint Specification Language (CCSL), is part of MARTE Profile but is not normative and not based on any existing language to let tool vendors choose their own technology. Our goal has been to use explicit keywords that denote usual concepts of the domain (periodic, sporadic, sampling...).

A comprehensive informal description of CCSL has previously been presented in [2] and a partial formal declarative description is available in [1]. Using a declarative mathematical description allows for being language independent. When constraints are not incompatible they should enforce a causal relationship between UML model elements and thus provide a support to build a real-time UML simulator. To implement CCSL and produce acceptable executions, i.e., compatible with all constraints, it may be interesting to transform it into equivalent formalisms that already benefit from analysis tools. After a brief overview of the semantics of a core CCSL constraint subset, this paper proposes several graphical UML-compatible possible representations of these constraints. These graphical representations are not part of MARTE yet and may be proposed to the second OMG Finalization Task Force for being integrated in next MARTE revision.

Section 2 starts with a brief overview of MARTE Time structure, the official OMG profile. Then, Section 3 describes main aspects of the non-normative constraint language CCSL. Section 4 compares three different visual representations of the constraints.

\section{MARTE Time subprofile}

\subsection{Time Structure}

A Clock is a 5 -tuple $\langle\mathcal{I}, \prec, \mathcal{D}, \lambda, u\rangle$ where $\mathcal{I}$ is a set of instants (possibly infinite), $\prec$ is a quasi-order relation on $\mathcal{I}$, named strict precedence, $\mathcal{D}$ is a set of labels, $\lambda: \mathcal{I} \rightarrow \mathcal{D}$ is a labeling function, $u$ is a symbol, standing for a unit. In this paper, we only consider the clock temporal structure (or pure clock), i.e., the ordered set $\langle\mathcal{I}, \prec\rangle$ and the values are never mentionned. $\prec$ is a total, irreflexive, and transitive binary relation on $\mathcal{I}$.

A discrete-time clock is a clock with a discrete set of instants $\mathcal{I}$. Since $\mathcal{I}$ is discrete, it can be indexed by natural numbers in a way that respects the ordering on $\mathcal{I}$ : let $\mathbb{N}^{\star}=\mathbb{N} \backslash\{0\}, \operatorname{idx}: \mathcal{I} \rightarrow \mathbb{N}^{\star}, \forall i \in \mathcal{I}, \operatorname{idx}(i)=k$ if and only if $i$ is the $k^{\text {th }}$ instant in $\mathcal{I}$. We restrict the discussion to discrete-time clocks and do not consider dense time at all. For all operators, we always assume that clocks are discrete, whereas these operators may have a more general semantics when applied to dense clocks.

For any discrete-time time structure $c=\left\langle\mathcal{I}_{c}, \prec_{c}\right\rangle, c[k]$ denotes the $k^{\text {th }}$ instant in $\mathcal{I}_{c}$ (i.e., $\left.k=\operatorname{idx}_{c}(c[k])\right)$. For any instant $i \in \mathcal{I}_{c},{ }^{\circ} i$ is the unique immediate predecessor of $i$ in $\mathcal{I}_{c}$ and $i^{\circ}$ is the unique immediate successor of $i$ in $\mathcal{I}_{c}$, if any. To simplify computations, we assume a virtual instant $c[0]$, so that $c[0] \equiv{ }^{\circ} c[1]$. Note, these definitions of predecessor and successor are only possible with a discrete structure.

A Time Structure is a pair $\langle C, \preccurlyeq\rangle$ where $C$ is a set of clocks, $\preccurlyeq$ is a binary relation on $\bigcup_{c \in C} \mathcal{I}_{c}$, named precedence. $\preccurlyeq$ is reflexive and transitive. From $\preccurlyeq$ we derive four new 
instant relations: Coincidence $(\equiv \triangleq \preccurlyeq \cap \succcurlyeq)$, Strict precedence $(\prec \triangleq \preccurlyeq \backslash \equiv)$, Independence $(\| \triangleq \preccurlyeq \cup \succcurlyeq)$, and Exclusion $(\# \triangleq \prec \cup \succ)$.

\subsection{UML Profile}

Figure 1 presents a simplified view of MARTE Time subprofile. The green elements are defined outside the profile itself but are useful to understand the profile. The stereotype ClockType extends the metaclass Class. It models classes of compatible clocks, i.e., clocks of the same nature (discrete or dense) and that use the same set of units. The stereotype Clock extends metaclasses InstanceSpecification and Property. It models a set of instants as defined in the previous subsection. Clocks can appear either in instance diagrams to represent a snapshot of a system at a given time or in composite structure diagrams to represent a family of possible behaviors. Starting from there, several identified model elements can be associated with one or several clocks using one the concrete stereotype that specializes the abstract stereotype TimedElement. This association with a clock gives the ability to the model element to embedd expressions or value specifications identifying precisely instants or durations. Having different reference clocks is very useful in distributed systems where different elements use different time bases. It is also useful in electronic design with manycore architectures or even monocore where several time domains are defined (main clock, bus clock...). Locally, it is often better to consider these time domains/bases (clocks) as independent so each part can be designed separately. However, when integrating it is required to understand the relations between these clocks to deal with inter-domain communications. Clock constraints have been defined with that objective. They make explicit relations amongst clocks. The stereotype ClockConstraint extends the metaclass Constraint and the Clock Constraint Specification Language (CCSL) gives a possible non-normative concrete syntax to specify constraints. CCSL is briefly introduced in the next section.

\section{Clock Constraints}

The Time structure defines relations between instants. However, a clock being an infinite set of instants it is neither realistic nor practical to specify constraints one by one. Based on instant constraints, it is easy to build more powerful relations that define infinitely many instant relations. We call these relations clock constraints. We can classify clock constraints into three families: coincident-based, precedence-based and mixed constraints.

\subsection{Coincidence-based constraints}

The coincidence-based clock constraints defines infinitely many coincidence instant relations. Most of the time, this kind of constraint defines a subclock from a given superclock, i.e., a clock less frequent. For instance we can select every third instant to create a periodic subclock of period 3. But as most clock constraints are relations, as opposed to functions, they can also do the contrary and define a superclock from one (or a set of) subclock(s). For example, to model a phase-lock loop (PLL) system, one may wish to oversample four times a given clock. The most frequently used constraint 


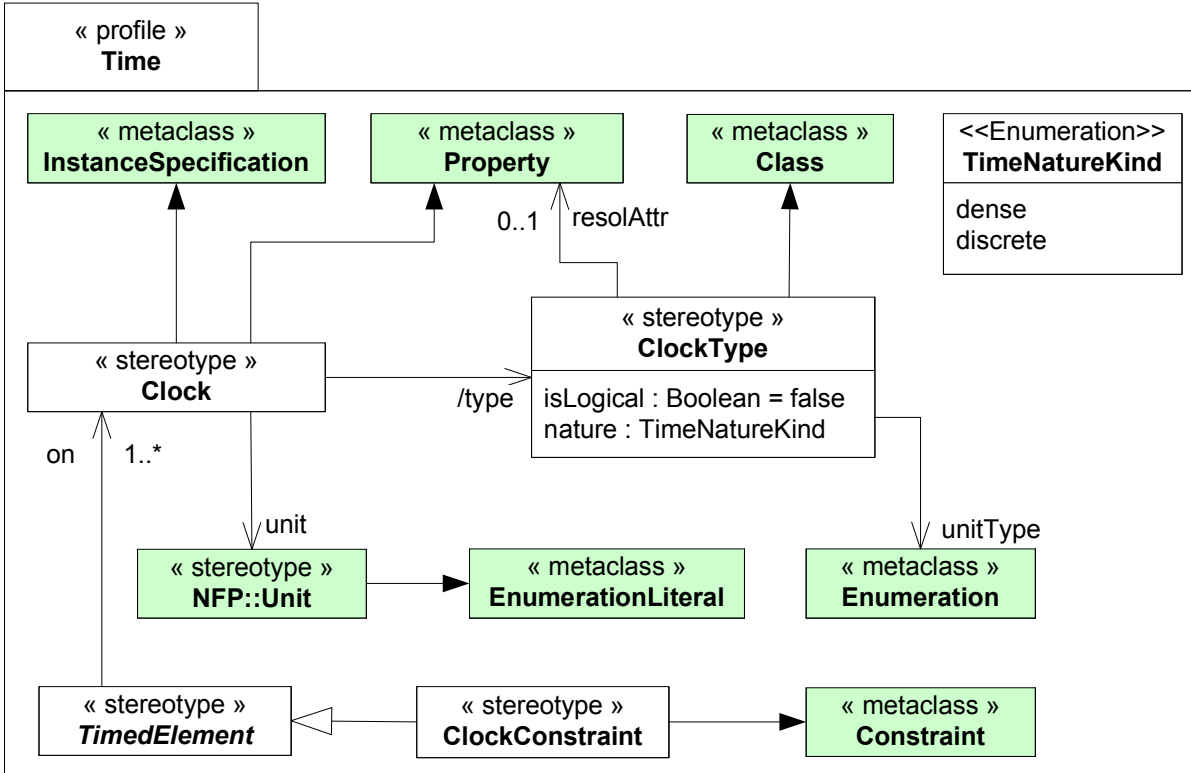

Fig. 1 Excerpt of MARTE Time subprofile

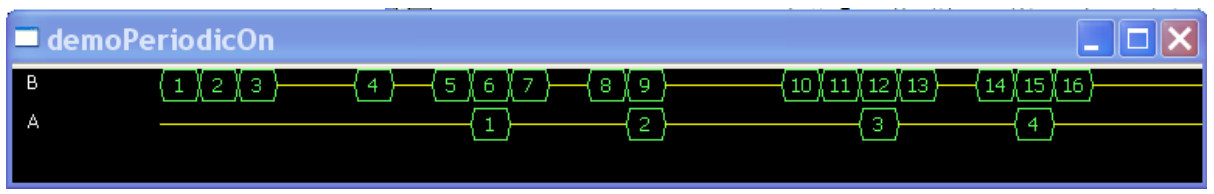

Fig. 2 A isPeriodicOn B period $=3$ offset $=5$

of this family is isPeriodicOn whose semantics is given in a mathematical declarative way by Eq. 1.

$$
\begin{array}{r}
A \text { isPeriodicOn } B \text { period }=P \text { offset }=\delta \\
\Longleftrightarrow \\
\left(\forall k \in \mathbb{N}^{\star}\right)(A[k] \equiv B[(k-1) * P+\delta+1])
\end{array}
$$

Figure 2 illustrates this relation with a period of 3 and an offset of 5 . This family describes synchronous relations inspired from operators defined in synchronous languages [3].

\subsection{Precedence-based constraints}

The precedence-based clock constraints define infinitely many precedence instant relations. They characterized asynchronous relations. The most frequently used constraint of this family is alternatesWith. The relation alternatesWith represents alternation between two clocks. A $\sim$ B means that each occurrence of $A$ is followed by an occurrence of 
$B$ before any other occurrences of $A$. The weak form of this relation allows the $i^{\text {th }}$ occurrence of $B$ to be simultaneous (coincident) with the $i^{\text {th }}$ occurrence of $A$, whereas the strict form requires $A$ and $B$ to be disjoint.

Typically, an asynchronous communication implies an alternation between sending and receiving. Let $A$ be the sender and $B$ the receiver. The data is received after having been sent. No other communication can start before the previous one completes. The weak form allows the sender to receive data simultaneously with the emission, but do not force the synchronization. The strict form is used to forbid instantaneous communications. The semantics of the strict form is given by Eq. 2 whereas the semantics of the weak form is given by Eq. 3 .

$A$ strictly alternatesWith $B$

$$
\begin{array}{r}
\Longleftrightarrow \\
\left(\forall k \in \mathbb{N}^{\star}\right)(A[k] \prec B[k] \prec A[k+1]) \\
A \text { alternatesWith } B \\
\Longleftrightarrow \\
\left(\forall k \in \mathbb{N}^{\star}\right)(A[k] \preccurlyeq B[k] \prec A[k+1])
\end{array}
$$

\subsection{Mixed constraints}

Mixed constraints combine both precedence and coincidence relations. There are useful when modeling communications from an asynchronous part of a design to a synchronous part. The most frequently used constraint of this family is sampledOn. The relation sampledOn represents sampling, it can be used to model time-triggered communications or for synchronizing asynchronous inputs. $A=B \downarrow C$ defines a subclock of $C$ that occurs only after an occurrence of $B$. The strict form of sampledOn does not instantaneously sample an occurrence of $B$ when it is synchronous with an occurrence of $C$. In that case, the sampling is postponed.

Figure 3 shows one possible scenario involving the clock relation sampledOn with both forms weak and strict. Signal $B$ counts its occurrences and signal $A$ contains the value actually sampled from $B$.

With both forms the first sample has the value 1 . However, with the weak form the first sample occurs on the first occurrence of $C$ whereas it occurs on the second occurrence of $C$ with the strict form. The second sample has the value 3 and the input 2 has been lost in both cases. The third sample occurs at the same time, whatever the form, but does not carry the same value in both cases. The semantics of the strict form is given by Eq. 4 whereas the semantics of the weak form is given by Eq. 5 .

$$
\begin{array}{r}
A=B \text { strictly sampledOn } C \\
\Longleftrightarrow \\
\left(\forall a \in \mathbb{N}^{\star}\right)\left(\exists b, c \in \mathbb{N}^{\star}\right) \\
((A[a] \equiv C[c]) \wedge(C[c-1] \preccurlyeq B[b] \prec C[c]))
\end{array}
$$




\section{demoSampledOn}

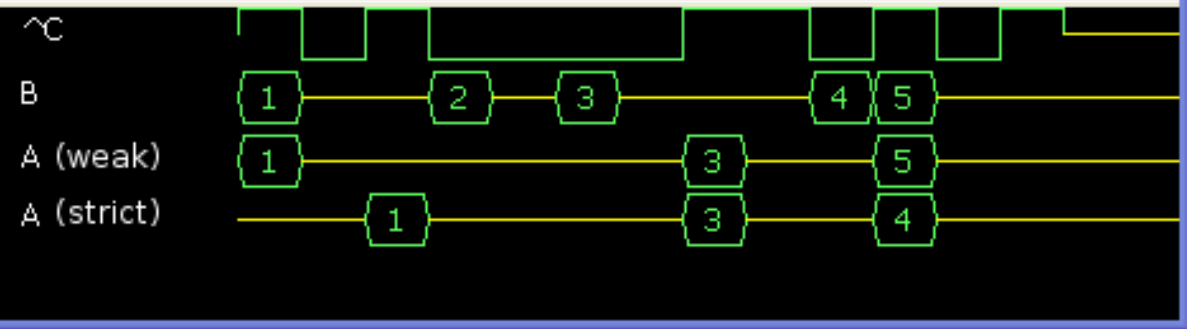

Fig. $3 \mathrm{~A}=\mathrm{B}$ sampledOn $\mathrm{C}$

$$
\begin{array}{r}
A=B \text { sampledOn } C(A=B \downarrow C) \\
\Longleftrightarrow \\
\left(\forall a \in \mathbb{N}^{\star}\right)\left(\exists b, c \in \mathbb{N}^{\star}\right) \\
((A[a] \equiv C[c]) \wedge(C[c-1] \prec B[b] \preccurlyeq C[c]))
\end{array}
$$

\section{Applying Constraints to UML models}

4.1 Using UML constraints

To apply these constraints, one must first create clocks and therefore clock types. Some very useful clock types are provided in MARTE library, like IdealClock that represents a perfect dense chronometric clock. Other clocks, with flaws like jitter, drift, can be derived from IdealClock or rather from one of its instances (a clock). The clock idealCIk is also provided in MARTE library and is an instance of IdealClock. idealClk can be discretized with a given resolution to build, for instance, a chronometric discrete clock whose frequency is $100 \mathrm{~Hz}$ (see Eq. 6).

$$
c_{100}=i d e a l C l k \text { discretizedBy } 0.01
$$

More generally, MARTE also provides a support to use logical clocks, i.e., clocks not directly related to the physical time. Anything that has to be compared (before, after or simultaneous) to something else can be considered as a logical clock. For instance, to give timing information about thread dispatch, a class Thread can be defined and the stereotype ClockType can be applied to it. Doing so, instances of Thread or properties/parts/ports of type Thread may become clocks. Note that, being a ClockType does not prevent from being something else, like a SchedulableResource, it only provides for a support to build clock constraints and express causality relations with other clocks.

Figure 4 illustrates such a case where two periodic threads $(t 1, t 3)$ are mixed with an aperiodic thread $(t 2)$. The two clock constraints make the two threads (clocks) periodic relative to clock $c_{100}$. The two threads are harmonic since they refer to the same clock with an offset 0 and the ratio of their period is an integer. Thread $t 2$ is 


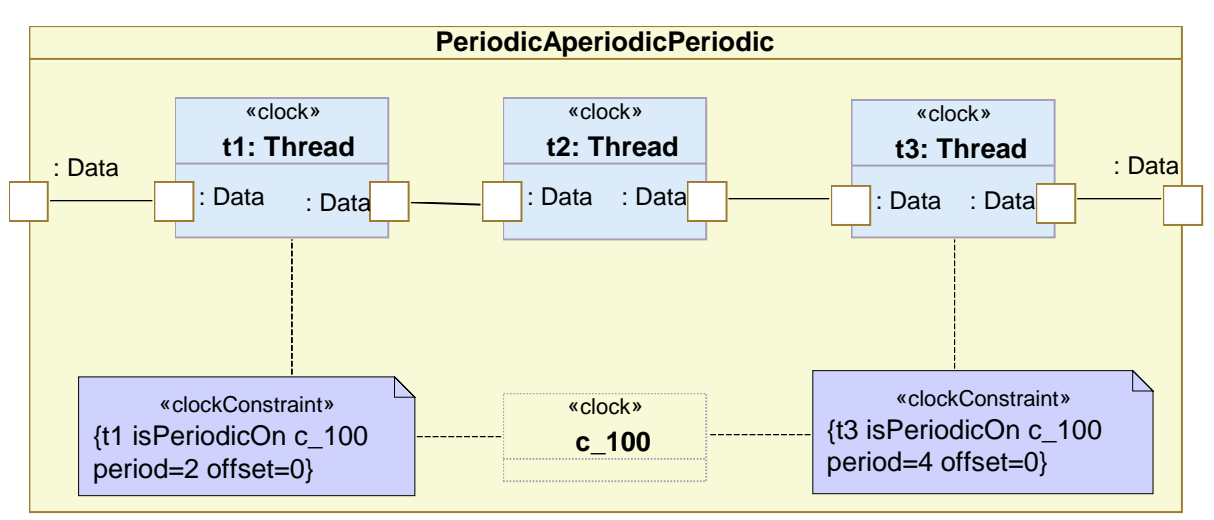

Fig. 4 Periodic threads with CCSL

aperiodic, no relation relates it to a clock. Clock clk is a shared clock (for instance, a chronometric discrete clock) and as such appears within a dashed rectangle. clk is not owned by the class PeriodicAperiodicPeriodic and could be used in another composite structure diagram.

However, a more obvious visual notation may be more appropriate. In particular, constraints are visually explicit when all the constrained elements appear on the same diagram. One purpose of the Time model is to bring consistency between different diagrams through clocks. Different model elements from different diagrams are connected together by clock constraints, which constrain them to behave in a consistent way. The two following subsections propose alternative notations where all clocks are shown on the same diagram.

\subsection{Using a CCSL-specific profile}

UML profile are a way to introduce new concepts but also to change the visual notation. Introducing a new visual language for clock constraints within MARTE is not practical because MARTE is very big and there are some many other matters to address. A solution can be to define aside, a CCSL-specific profile. Each often used CCSL constraint can have its own stereotype associated with a specific icon. The most appropriate meta-class to represent CCSL constraint is probably the metaclass Dependency.

Figure 5 shows a possible profile that could be defined to ease the use of CCSL constraints. The left-hand side part of the figure shows the actual profile. The righthand side part of the figure shows another composite structure diagram about the same three-thread example. In this example, the diagram represents three new clocks (step 1 , step 2 and step 3 ) that are the actual behaviors (subprograms) executed by the threads. These clocks could also have been shown on the previous diagram but this would have resulted in a very heavy construct. Using the defined stereotypes and their icons brings simplicity even though the semantics is exactly the same.

The relation alternatesWith from the threads to their assigned subprogram denotes that every time the thread is dispatched, the subprogram must execute. Additionnally, the subprogram cannot be executed another time before the completion of the previous execution. 


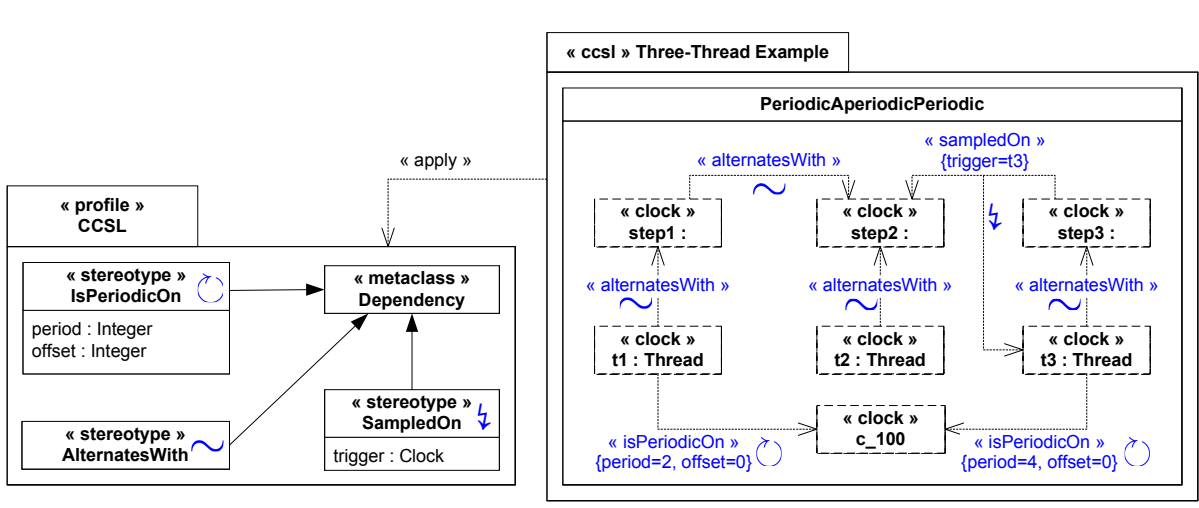

Fig. 5 Using a CCSL-specific profile

From step1 to step2, there is also a relation alternatesWith since there is an asynchronous communication. Whenever step1 completes, step2 takes its output data and executes.

Finally, the ternary relation involving step2, step3 and $\mathrm{t} 3$ denotes the synchronization of step2 output according to the clock t3. Every time $\mathrm{t} 3$ is dispatched, whatever the current status of step2 output, the data available is sampled and step3 executes using this sample. Obviously, this may lead to data loss, if the sampling rate is too low or to the use of the sample data in multiple executions of step3 if the sampling rate is too high.

This representation requires to define a new profile aside MARTE. In this profile, each CCSL constraint has its own stereotype and its properties are determined according to CCSL grammar. An alternative to the creation of another profile is to use parametric diagrams as described in the following subsection.

\subsection{Using SysML parametric diagrams}

The UML profile for System Engineering (SysML) [8] is an adopted OMG Specification to be used at the system level. They have selected a subset of UML constructs called UML4SysML and they provide few new extensions amongst which Refinement and Parametric diagrams. The former helps making explicit system-level requirements and tracing their proposed implementations. The latter should be used to represent noncausal relations among values of the system and possibly making explicit within the model, physical laws required for the design.

So, we can use this SysML construct to represent laws related to time, whether physical or logical. SysML recommends building a new "Constraint Block" for each new law and then use it in so-called parametric diagrams to apply this law to relevant design values. In our case, we have a small number of identified relations among logical clocks. Consequently, we can easily construct a library of CCSL-specific constraint block.

Figure 6 illustrates the same example using SysML constraint blocks and parametric diagrams.

The left-hand side part is an excerpt of the library. Three constraint blocks (Periodic, Alternation, Sampling) have been defined for each of the three CCSL relations introduced previously. Each constraint block has two compartments. The bottom one, called 

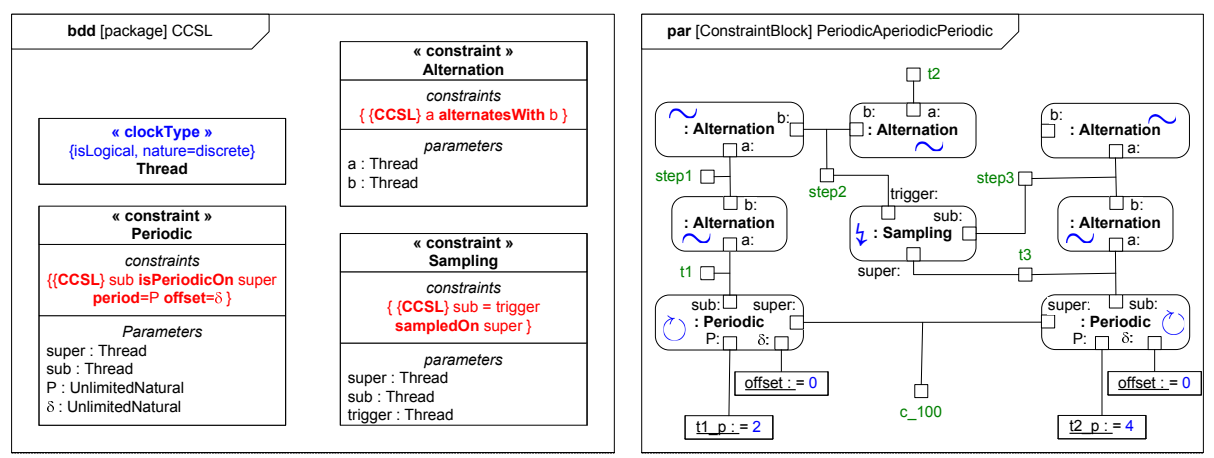

Fig. 6 Using SysML parametric diagrams

parameters contains typed formal parameters. The upper compartment, called constraints, contains the constraint itself that applies on the parameters. In our case, the constraint is defined in CCSL. However, this library is built once and for all, so end-users need not being entirely familiar with the concrete syntax and only need to be familiar with underlying concepts.

The right-hand side part presents the three-thread example as a SysML parametric diagram. In such a diagram, boxes are properties extracted from the model. Some of the properties are clocks $\left(t 1\right.$, step $1 \ldots$ ), some others have integer values (offset, $t 1 \_p \ldots$... These properties may come from different diagrams and different blocks. The rounded rectangles are usages of constraint blocks. Their ports, which represent parameters, are connected with properties using non-causal connectors. Being non-causal means that there is no input or output, whichever value is known causes the other to update. For instance, considering Alternation, if $b$ is known, one can deduce (partially) $a$ but if $a$ is known, then one can deduce (partially) $b$.

\section{5 conclusion}

The paper has presented some of the clock constraint relations introduced by MARTE Time model together with their formal semantics. These clock constraints brings consistency in the timing information of UML models. Introducing formal models in UML models is a problem. OMG Specifications are usually big and are not the right place to put formal specification. Leaving the formal semantics outside the specification leaves the interpretation to tools, possibly having different interpretations in different tools.

With CCSL, we rely UML constraints. However, constraints are not the best visual representation. We propose here two visual alternative notations that result in more compact diagrams. These two solutions are not specific to CCSL and could be easily extended to other constraint-based languages like OCL. The limitation comes from finding the right icon or shape that makes immediately the link with the constraint. Our first proposition implies the creation of a CCSL-specific profile. The second one relies on SysML parametric diagrams. It is interesting to see that all the proposed notations are composite structure diagrams or derived notations.

These two notations attempts to replace CCSL concrete syntax by a visual representation and to allow a representation of otherwise scattered timing information in a single diagram. 
The next step is to provide analysis support for such diagrams. We already have implemented an Eclipse-based simulator for CCSL constraints [1] and we are trying to integrate it with parametric diagram to get a direct visual feedback of a simulation run. We have also made some progress to transform CCSL constraints into other formalisms, like Signal or Petri nets, that have their own analysis techniques and tools [5].

Acknowledgements Some UML diagrams have been built using Papyrus, an open-source tool available at http://www.papyrusuml.org.

\section{References}

1. André, C., Mallet, F.: Clock constraints in UML/MARTE. Research Report 6540, INRIA (2008). URL http://hal.inria.fr/inria-00280941/en/

2. André, C., Mallet, F., de Simone, R.: Modeling time(s). In: G. Engels, B. Opdyke, D.C. Schmidt, F. Weil (eds.) MoDELS, Lecture Notes in Computer Science, vol. 4735, pp. 559573. Springer (2007)

3. Benveniste, Caspi, Edwards, Halbwachs, Guernic, L., de Simone: The synchronous languages twelve years later. Proceedings of the IEEE 91(1) (2003)

4. Lee, E.A., Sangiovanni-Vincentelli, A.L.: A framework for comparing models of computation. IEEE Transactions on Computer-Aided Design of Integrated Circuits and Systems $\mathbf{1 7}(12), 1217-1229$ (1998)

5. Mallet, F., André, C.: UML/MARTE CCSL, signal and petri nets. Research Report 6545, INRIA (2008). URL https://hal.inria.fr/inria-00283077

6. Object Management Group: Unified Modeling Language, Superstructure (2007). Version 2.1 .2 formal/2007-11-02

7. Object Management Group: UML Profile for MARTE, beta 2 (2008). OMG document number: ptc/08-07-09

8. OMG: Systems Modeling Language (SysML) Specification 1.1 (2008). OMG document number: ptc/08-05-17 